\title{
Recognition of MQAM signals in the Aeronautical communication
}

$$
\text { Huang Guo-qing }{ }^{1,2, a} \text {, Zhang Cheng-gen }{ }^{1, b} \text { and Yuan Hong-bo }{ }^{2, c}
$$

\author{
${ }^{1}$ School of Information Engineering, Zhengzhou University, Zhengzhou 450001, China \\ ${ }^{2}$ Department of Aeronautic Electronic Engineering, The First Aeronautical College of Air Force, \\ Xinyang, 46400, China
}

ahguoqing@163.com, bzhangchenggenzcg@163.com, chongboyuan_76@163.com

Keyword: Modulation recognition; Aeronautical channel; High order cumulants; Receive diversity

\begin{abstract}
For the complex recognition of signals in the aeronautical communication, the recognition of the star-type MQAM signals is studied under the aeronautical channel. The different scenarios of the aeronautical channel are analyzed and the reasonable channel model is established. The high order cumulant recognition algorithm which applies to the aeronautical channel is proposed, the invariant classification characteristics of modulation signals are constructed by this algorithm, and this algorithm is improved by using the receive diversity technique. Simulation results show that this algorithm significantly improves the correct recognition rate of the received signals has the good anti-fading and anti-noise performance and meets the requirements of the modulation recognition of MQAM signals under the aeronautical channel.
\end{abstract}

\section{Introduction}

The modulation recognition of communication signals is the process in which the modulation type of signals is correctly decided and some modulation parameters are estimated in the case of the unknown modulation information or the given information. These methods are generally divided into two categories: the first category is the maximum likelihood method based on hypothesis tests while the second is the pattern recognition method based on feature extraction $[1,2]$.

The MQAM signal is an important modulation method and is widely used in modern digital communication system; therefore the research of its recognition is of important significance. In $[3,4]$, the high order cumulant algorithm is used to recognize the MQAM signals. In addition, the MIMO system pointed out a new direction for the modulation recognition researches [5]. [6] which used cumulant characteristics of signals to recognize MQAM signals in the MIMO system.

The modulation recognition of signals is studied under aeronautical channel whose band is the ultra-short wave band. The aeronautical channel is a special class of time-varying channel with different characteristics under different motion states of the aircraft. The following scenarios are analyzed: taxi scenario, takeoff and arrival scenario and en-route scenarios [7]. Assuming that the fading channel has been estimated and compensated, with receive diversity technique, the star-type MQAM signals are recognized by using the high order cumulant algorithm.

\section{Aeronautical Channel Model}

The different conditions of an aircraft lead to different channel scenarios during flight. These scenarios are determined by the type of fade, the Doppler and delay power spectra. In general, several scenarios are as follows: en-route scenario, taxi scenario, takeoff and arrival scenario etc. The channel of these scenarios consists of a LOS path and some scattered paths. Assuming that the lowest angle and the highest angle of arrival of antenna beams are expressed by $\alpha_{L}$ and $\alpha_{H}$ respectively, the beamwidth [7] is defined as $\alpha_{b}=\alpha_{H}-\alpha_{L}$. The different beamwidths lead to the different forms of the Doppler power spectrum [10]. The different directions of the LOS path result in the different Doppler frequency shifts [7].

In summary, the channel model of the aircraft different states is expressed as a Rice model. 


$$
h(k)=a \exp \left(\mathrm{j} 2 \pi f_{D_{\text {Los }}} k T_{\text {sample }}\right)+\lim _{N \rightarrow \infty} \frac{b}{\sqrt{N}} \sum_{n=1}^{N} \exp \left(j \varphi_{n}\right) \exp \left(j 2 \pi f_{D_{n}} k T_{\text {sample }}\right)
$$

Where $a \in R$ is the amplitude of the LOS path and $f_{D_{\mathrm{Los}}}$ is the Doppler frequency of the LOS path, $k$ denotes a time index, $T_{\text {sample }}$ is a sampling period, $b^{2} \in R$ is the variance of scattered components, $N$ denotes the number of scattered path. The random numbers $\varphi_{n}, f_{D_{n}}$ and $\tau_{n}$ denote the random phase, Doppler shift and random delay of the nth path respectively.

\section{Recognition of MQAM signals based on HOC}

A. Algorithm Derivation

Assuming that MQAM signals have been preprocessed, such as down-converting, intermediate frequency filtering and carrier frequency synchronizing, etc. At the output of the receiver matched filter the baseband signals can be written as

$$
y(k)=\sqrt{\rho} x(k) \cdot h(k)+w(k), k=1, \cdots, L .
$$

where $h(k)$ is shown in (1), since the $h(k)$ value is the complex value, so expression (1) can be simplified as $h(k)=h_{0} e^{j \psi_{0}}+\sum_{n=1}^{N} h_{n} e^{j \psi_{n}}$, where $h_{n}$ and $\psi_{n}(n=0,1, \cdots, N)$ denote the amplitude and the phase of different paths. $x(k)$ denote the MQAM equivalent baseband signal sequence, which is a zero-mean independent and identically distributed random variable, $x(k)=\sqrt{A} e^{j \theta_{k}} a_{k}, a_{k} e^{j \theta_{k}}$ are the interesting symbol sequence of the received signal whose average power is normalized. $a_{k}$ and $\theta_{k}$ are the amplitude and phase of the signal respectively [8]. A is the average power of the signal, $w(k)$ is the additive white complex Gaussian noise with zero mean and variance $\sigma^{2}$, and $x(k)$ and $w(k)$ are independent. $L$ is the length of the sampled data.

Referring the definition of the fourth-order cumulate [3], the cumulant values of the signal $x(k)$ are gotten and shown in Table 1.

Table 1 Fourth-order cumulants of star-type MQAM signals

\begin{tabular}{l|l|l|l}
\hline & $C_{x, 42}$ & $C_{x, 41}$ & $C_{x, 40}$ \\
\hline 2QAM & $-2 A^{2}$ & $-2 A^{2} e^{j 2 \theta_{K}}$ & $-2 A^{2} e^{j 4 \theta_{k}}$ \\
\hline 4QAM & $-A^{2}$ & 0 & $A^{2} e^{j 4 \theta_{k}}$ \\
\hline 8QAM & $-0.51 A^{2}$ & 0 & $1.49 A^{2} e^{j 4 \theta_{k}}$ \\
\hline 16QAM & $-0.72 A^{2}$ & 0 & 0 \\
\hline
\end{tabular}

Then the invariant classification characteristic vector is constructed.

$$
F_{x}=\left[f_{x 1}, f_{x 2}\right]=\left[\frac{\left|C_{x, 40}\right|}{\left|C_{x, 42}\right|}, \frac{\left|C_{x, 41}\right|}{\left|C_{x, 42}\right|}\right]
$$

For the different star-type MQAM signals, the invariant classification characteristic vectors are given by.

$$
F_{x}= \begin{cases}{[1,1]} & 2 \mathrm{QAM} \\ {[1,0]} & 4 \mathrm{QAM} \\ {[2.92,0]} & \text { 8QAM } \\ {[0,0]} & 16 \mathrm{QAM}\end{cases}
$$

The fourth-order cumulant value of Gaussian noise is zero, and noise and signal are mutually independent, the following expressions can be obtained according to the cumulant properties [7].

$$
\begin{gathered}
C_{y, 40}=\operatorname{cum}[y(k), y(k), y(k), y(k)]=\rho^{2} A^{2} C_{x, 40} \sum_{n=0}^{N} h_{n}^{4} e^{j 4 \psi_{n}} \\
C_{y, 41}=\operatorname{cum}\left[y(k), y(k), y(k), y(k)^{*}\right]=\rho^{2} A^{2} C_{x, 41} \sum_{n=0}^{N} h_{n}^{4} e^{j 2 \psi_{n}}
\end{gathered}
$$




$$
C_{y, 42}=\operatorname{cum}\left[y(k), y(k), y(k)^{*}, y(k)^{*}\right]=\rho^{2} A^{2} C_{x, 42} \sum_{n=0}^{N} h_{n}^{4}
$$

The classification characteristic vector is constructed again based on (3) and (4):

where

$$
F_{y}=\left[f_{y 1}, f_{y 2}\right]=\left[\frac{\left|C_{y, 40}\right|}{\left|C_{y, 42}\right|}, \frac{\left|C_{y, 41}\right|}{\left|C_{y, 42}\right|}\right]=\left[\eta_{1} \frac{\left|C_{x, 40}\right|}{\left|C_{x, 42}\right|}, \eta_{2} \frac{\left|C_{x, 41}\right|}{\left|C_{x, 42}\right|}\right] .
$$

$$
\eta_{1}=\left|\sum_{n=0}^{N} h_{n}^{4} e^{j 4 \psi_{n}} / \sum_{n=0}^{N} h_{n}^{4}\right|, \quad \eta_{2}=\left|\sum_{n=0}^{N} h_{n}^{4} e^{j 2 \psi_{n}} / \sum_{n=0}^{N} h_{n}^{4}\right| .
$$

Next, we prove the classification characteristic vector is invariant for the aeronautical channel parameters, namely to prove $F_{y}=F_{x}$.

This problem is proved based on the simplified aeronautical channel model. Let

$$
r(l)=\sum_{n=0}^{N} h_{n}^{4} e^{j l \psi_{n}}=h_{0}^{4} e^{j l \psi_{0}}+\sum_{n=1}^{N} h_{n}^{4} e^{j l \psi_{n}}=h_{0}^{4} e^{j l \psi_{0}}+H_{l} e^{j \phi_{l}}, \quad l=0,2,4 .
$$

In (7) $H_{l}$ and $\phi_{l}$ represent the amplitude and the phase of the non-line-of-sight path respectively. Let $h_{0}=\max \left[h_{n}, n=0,1, \cdots . N\right]$, and not consider the impact of the phase, then $h_{0}^{4} \gg H_{l}$, so $r(l)$ is obtain:

$$
r(l) \approx h_{0}^{4} e^{j l \psi_{0}}, \quad l=0,2,4 .
$$

From (6) and (8), $\eta_{1} \approx \eta_{2} \approx 1$ is obtained, therefore, $F_{y}=F_{x}$, which shows $F_{y}$ is invariant for the aeronautical channel parameters, so it is used as the recognition characteristic of received signals.

The decision criterion of the star-type MQAM signals' recognition is referred in [8].

B. Receive Diversity Technique

Diversity is an important technique, which can compensate the fading channel and improve the quality of the received signal, so it is used to process the received signal. In order to recognize star-type MQAM signals well, the channel is estimated and compensated, then, signals are received by using the receive diversity technique.

For a multiple-input multiple-output (MIMO) system, assuming that there are $M$ transmitting antennas and $N$ receiving antennas in the system. The received signals are given by [6]:

$$
\boldsymbol{Y}=\sqrt{\rho} \boldsymbol{X} \boldsymbol{H}+\boldsymbol{W} .
$$

where $\boldsymbol{Y}=\left[y_{1}, \cdots, y_{N}\right]$ is the $(1 \times N)$ received signal vector, $\boldsymbol{X}=\left[x_{1}, x_{2}, \cdots, x_{M}\right]$ is the $(1 \times M)$ transmitted signal vector, and $\boldsymbol{W}=\left[w_{1}, w_{2}, \cdots, w_{N}\right]$ is the $(1 \times N)$ vector corresponding to Gaussian noise with its components obeying complex Gaussian distribution, $\boldsymbol{H}=\left[h_{1}, h_{2}, \cdots, h_{N}\right]$ is the $(M \times N)$ complex matrix of the MIMO channel, $h_{i}=\left[h_{1 i}, h_{2 i}, \cdots, h_{M i}\right]^{\mathrm{T}}$ is the $(M \times 1)$ vector, $i=1,2, \cdots, N, \rho$ is the signal-to-noise ratio.

Scheme 1: when $M$ and $N$ are one respectively, MIMO becomes a single-input single-output (SISO) system, the received signals are given by:

$$
y=\sqrt{\rho} x h+w .
$$

Under the ideal channel estimation, (10) can be transformed into (11)

$$
\hat{x}=\frac{h^{\mathrm{H}} y}{\sqrt{\rho}|h|^{2}}=x+\frac{h^{\mathrm{H}} w}{\sqrt{\rho}|h|^{2}} .
$$

$\hat{x}$ is regarded as the final received signal $\hat{y}$ in (11), then $\hat{y}$ is recognized.

Scheme 2: when $M=1$ and $N=3$ are true, (9) is processed to become (12) directly.

$$
\hat{\boldsymbol{X}}=\boldsymbol{Y} \boldsymbol{H}^{\mathrm{H}}\left(\boldsymbol{H H}^{\mathrm{H}}\right)^{-1}=\boldsymbol{X}+\boldsymbol{W} \boldsymbol{H}^{\mathrm{H}}\left(\boldsymbol{H H}^{\mathrm{H}}\right)^{-1}
$$

Take $\hat{\boldsymbol{X}}$ as the final received signal $\hat{\boldsymbol{Y}}=\hat{\boldsymbol{X}}$, then $\hat{\boldsymbol{Y}}$ is recognized.

\section{Simulation and Results Analysis}

The parameter values of every aeronautical scenario are listed in Table 2 [9]. 
Tab.2 Parameter values of every scenario

\begin{tabular}{l|c|c|c|c}
\hline & Taxi scenario & $\begin{array}{c}\text { Takeoff and Arrival } \\
\text { scenario }\end{array}$ & $\begin{array}{c}\text { En-Route(ground-air) } \\
\text { scenario }\end{array}$ & $\begin{array}{c}\text { En-Route(air-air) } \\
\text { scenario }\end{array}$ \\
\hline Aircraft velocity[m/s] & 15 & 150 & 440 & 620 \\
\hline Rice factor $K_{\text {Rice }}[\mathrm{dB}]$ & 6.9 & 15 & 15 & 15 \\
\hline$f_{D_{\text {Los }}} / f_{D_{\max }}$ factor & 0.7 & 1 & 1 & $178.25^{\circ}$ \\
\hline Arrival angle $\alpha_{L}$ of beam & $0^{\circ}$ & $-90^{\circ}$ & $178.25^{\circ}$ & $181.75^{\circ}$ \\
\hline Exit angle $\alpha_{\boldsymbol{H}}$ of beam & $360^{\circ}$ & $90^{\circ}$ & $181.75^{\circ}$ & 1 \\
\hline
\end{tabular}

In addition, let the carrier frequency be $200 \mathrm{MHz}$, symbol period be $2 \times 10^{-5} \mathrm{~s}$, the number of scattered path be 20, then give 500 Monte Carlo experiments for every QAM signals independently. Only list the simulation results figures of en-route (air-air) and en-route (ground-air) scenarios.

(1) Based on scheme 1, the simulation results of en-route (air-air) and en-route (ground-air) scenarios are shown in Figure 1:

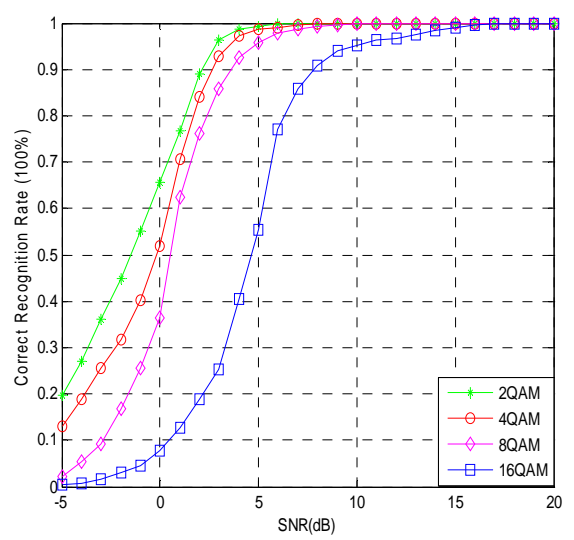

(a) en-route(ground-air) scenario modulation recognition performance curve

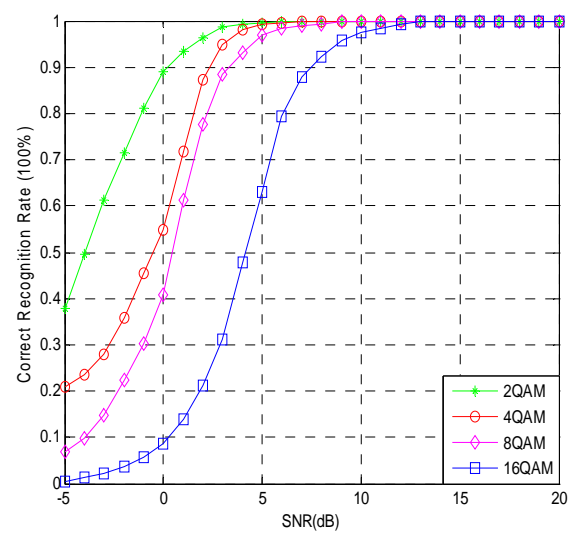

(b) en-route(air-air) scenario modulation recognition performance curve

Figure 1 the modulation recognition performance curve of MQAM signals in aeronautical channel when $\mathrm{N}=1$

Figure 1 and the simulation figures of taxi and takeoff and arrival scenarios show that the recognition performance of en-route scenario is better than that of taxi and takeoff and arrival scenarios. In addition, the channel compensation can reduce the channel fade, however, the recognition performance of taxi scenario is still poor due to multipath fade, Doppler frequency shift and Rice factor, and it is very difficult to achieve $100 \%$ correct recognition rate at $20 \mathrm{~dB}$. Because 8QAM and 16QAM signals are sensitive to the channel fade and signal-to-noise ratio, so their recognition performance is poorer than that of 2QAM and 4QAM signals, and their recognition rate will be even lower in the case of small Rice factor.

(2) Based on scheme 2, the simulation results of en-route (air-air) and en-route (ground-air) scenarios are shown in Figure 2:

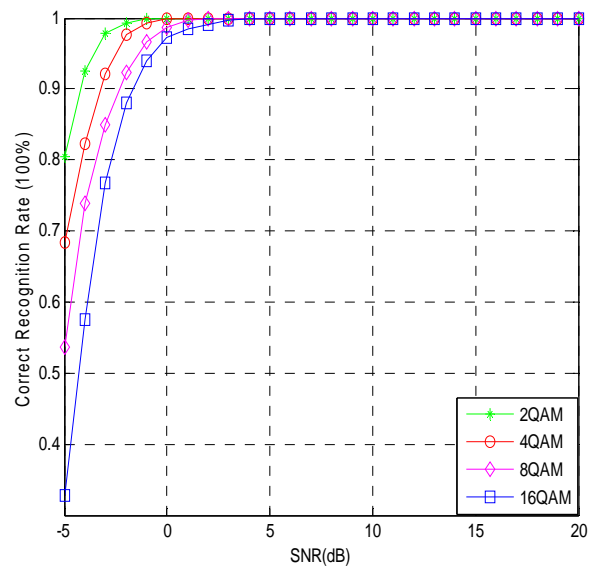

(a) en-route(ground-air) scenario modulation recognition performance curve

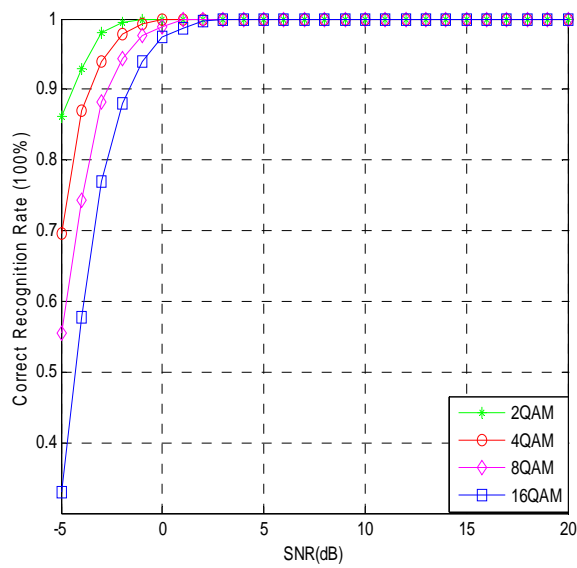

(b) en-route(air-air) scenario modulation recognition performance curve

Figure 2 the modulation recognition performance curve of MQAM signals in aeronautical channel when $\mathrm{N}=3$ 
At the receiver, the receive diversity technique and the method of the maximum ratio combining are used to process the received signals. The simulation results show that the quality of received signals has been effectively improved, multipath fade and Doppler frequency shift are compensated better, the correct recognition rate of the four types of signals has been significantly improved under every scenario, especially taxi scenario and 8QAM and 16QAM signals that are sensitive to the channel fade and signal-to-noise ratio. In addition, the recognition rate of signals is higher at the negative SNR, the completely correct recognition is achieved when SNR reaches $5 \mathrm{~dB}$. In short, the quality and the correct recognition rate of the received signal have been effectively improved by using the receive diversity technique at the receiver, which is able to meet the requirements of the modulation recognition of MQAM signals under the aeronautical channels.

\section{Conclusion}

The recognition of the star-type MQAM signals is studied under the aeronautical channel. The different scenarios of this channel are analyzed and a reasonable channel model is established. The receive diversity technique is used to process the received signal before the recognition, which can reduce the channel fade and Doppler frequency shift and improve the quality of the received signal. Finally, the high order cumulant recognition algorithm is used to construct the invariant classification characteristic of modulation signals and the star-type MQAM signals are recognized correctly. The simulation results show that this algorithm has the good anti-noise performance, and then the receive diversity technique is used in this process, which not only can improve the quality of the received signals, but also significantly improve the recognition rate of signals. This method can achieve the recognition of signals under the aeronautical channels.

\section{References}

[1] O. Dobre, A. Abdi, Y. Bar-Ness, and W. Su, "Survey of automatic modulation classification techniques: classical approaches and new trends,” IET Commun, vol. 1, No. 2, pp. 137-156, 2007.

[2] J. L. Xu, W. Su, and M. C. Zhou, "Likelihood ratio approaches to automatic modulation classification,” IEEE Trans. Systems, Man, and Cybernetics, vol. 41, pp. 455-469, July 2011.

[3] A. Swami and B. M. Sadler, "Hierarchical digital modulation classification using cumulants," IEEE Trans. Commun, vol. 48, No. 3, pp. 416-429, March 2000.

[4] W. D. Chen and S. Q. Yang, "Recursive Classification of MQAM signals based on higher order cumulants,” Journal of Electronics, vol. 19, No. 3, pp. 270-274, July 2002.

[5] V. Choqueuse, S. Azou, K. Yao, L. Collin and G. Burel, "Blind modulation recognition for MIMO systems,” J. ATM Bucharest, vol. XIX, No. 2, pp. 183-196, Jun 2009.

[6] K. Hassan, I. Dayoub, W. Hamouda, C. N. Nzéza, and M. Berbineau, "Blind Digital Modulation Identification for Special-Correlated MIMO Systems,” IEEE Trans. Wireless Communications, vol.11, No. 2, pp. 683-693, February 2012.

[7] E. Haas, “Aeronautical Channel Modeling,” IEEE Trans. Vehicular Technology, vol. 51, No. 2, pp. 254-264, March 2002.

[8] G. HAN, “The Blind Detection Technology Research of Signal in Adaptive Single Carrier, Multi-carrier Modulation,” Xi'an University of Electronic Science and Technology, 2003.

[9] O. A. Dobre, A. Abdi, Y. Bar-Ness, and W. Su, "Selection combining for modulation recognition in fading channels,” Proc IEEE MILCOM, 2005, pp. 2499-2505. 